\title{
Aconselhamento do usuário na realização do teste rápido Anti-HIV: Relato de acadêmicas de enfermagem
}

\author{
User advice on the realization of these fast Anti-HIV: Relating to an experience of nursing \\ academics consejos
}

Al usuario para realizar la prueba rápida Anti-VIH: Informar la experiencia de los académicos de enfermeira

Recebido: 25/05/2021 | Revisado: 02/06/2021 | Aceito: 10/06/2021 | Publicado: 23/06/2021

Larissa Scheeren Thomas
ORCID: https://orcid.org/0000-0002-5890-8105
Secretaria de Saúde de Florianópolis, Brasil
E-mail: lari_scheeren_thomas@ hotmail.com
Karen Pietrowski
ORCID: https://orcid.org/0000-0002-4513-0605
Associação Hospital de Caridade Santo Ângelo, Brasil
E-mail: karen.pietrowski@ outlook.com
Nadine Both da Silva
ORCID: https://orcid.org/0000-0002-7416-5751
Universidade Feevale, Brasil
E-mail: nadine.both@hotmail.com
Silvia dos Reis Feller
Faculdade UNIMED, Brasil
ORCID: https://orcid.org/0000-0002-8183-0174
E-mail: silvia.feller@yahoo.com.br
Lilian Zielke Hesler
ORCID: https://orcid.org/0000-0001-9363-2709
E-mail: lilianhesler@ @san.uri.br

\begin{abstract}
Resumo
Objetivo: relatar a experiência de acadêmicas de enfermagem frente à realização de testes rápidos anti-HIV, assim como, o enfrentamento dos resultados e aconselhamento do usuário. Metodologia: trata-se de um estudo descritivo com abordagem qualitativa, do tipo relato de experiência, vivenciado por quatro discentes e uma docente, durante as atividades práticas, no mês de abril de 2019. Relato de experiência: foram realizados seis testes rápidos anti-HIV, com um resultado positivo. Para a realização dos testes rápidos torna-se necessária a execução de técnica correta, o uso de Equipamentos de Proteção Individual (EPI) indispensáveis em qualquer assistência, boa comunicação entre os profissionais da equipe e profissionais da rede, bem como, um aconselhamento eficiente pré e pós-teste. A implementação, divulgação e realização dos testes rápidos na população, acabou tendo um propósito de orientação e acompanhamento do usuário, empoderando-o a ser o protagonista de sua saúde, apesar desta ser ainda uma deficiência na assistência. Conclusão: a vivência de um resultado positivo provocou mudança de visão e pensamentos, onde percebeu-se que há a necessidade de um trabalho em rede, com a disponibilidade de múltiplos profissionais para um cuidado integral e efetivo.
\end{abstract}

Palavras-chave: Enfermagem; Infecções por HIV; Aconselhamento.

\begin{abstract}
Objective: experience report of nursing students regarding the performance of rapid anti-HIV tests, as well as the confrontation of results and user counseling. Methodology: this is a descriptive study with a qualitative approach, of the type of experience report, lived by four students and one teacher, during the practical activities, in April 2019. Results and discussion: six rapid anti- HIV, with a positive result. For the performance of rapid tests it is necessary to perform the correct technique, the use of Personal Protective Equipment (PPE) indispensable in any assistance, good communication between team professionals and network professionals, as well as advice efficient pre and post-test. The dissemination, dissemination and performance of rapid tests in the population, ended up having a purpose of guidance and monitoring of the user, empowering him to be the protagonist of his health, despite this being still a deficiency in assistance. Conclusion: the experience of a positive result caused a change of vision and thoughts, where it is expected that there is a need for networking, with the availability of multiple professionals for comprehensive and effective care.
\end{abstract}


Key words: Nursing; HIV infections; Counseling.

\section{Resumen}

Objetivo: relato de experiencia de estudiantes de enfermería sobre la realización de pruebas rápidas anti-VIH, así como el enfrentamiento de resultados y asesoramiento a usuarios. Metodología: se trata de un estudio descriptivo con enfoque cualitativo, del tipo de relato de experiencia, vivido por cuatro alumnos y un docente, durante las actividades prácticas, en abril de 2019. Resultados y Discusión: seis anti- VIH, con resultado positivo. Para la realización de pruebas rápidas es necesario realizar la técnica correcta, el uso de Equipos de Protección Individual (EPI) indispensable en cualquier asistencia, buena comunicación entre los profesionales del equipo y los profesionales de la red, así como el asesoramiento prueba previa y posterior eficiente. La difusión, difusión y realización de pruebas rápidas en la población, terminó teniendo una finalidad de orientación y seguimiento del usuario, empoderándolo para ser el protagonista de su salud, a pesar de que esta sigue siendo una deficiencia en la asistencia. Conclusión: la experiencia de un resultado positivo provocó un cambio de visión y pensamiento, donde se espera que exista una necesidad de trabajo en red, con la disponibilidad de múltiples profesionales para una atención integral y eficaz.

Palabras clave: Enfermería; Infecciones por VIH; Consejería.

\section{Introdução}

O Vírus da Imunodeficiência Humana (HIV) se destaca por ser uma pandemia devido ao grande número de casos no mundo todo, causando assim uma preocupação mundial. Caracteriza-se pelas consequências que pode causar na vida do portador, onde pode gerar outras doenças oportunistas e caso não haja tratamento evoluir para a Síndrome da Imunodeficiência Adquirida (AIDS) (Silva, Júnior \& Rodrigues, 2014).

O Teste rápido, que é uma das técnicas de diagnóstico para o HIV de relevância nas unidades de Atenção Primária a Saúde (APS), com metodologia que permite a rápida detecção de anticorpos, em até 30 minutos, possui baixo custo e de fácil aplicação e interpretação, devendo ser realizado por profissionais devidamente capacitados (Ministério da Saúde, 2016). A testagem para o HIV tem como objetivo, um diagnóstico da infecção de melhor qualidade, uma vez que, é eficaz e de rápida conclusão, e assim, possibilita um tratamento precoce e adequado, evitando o desenvolvimento da infecção pelo vírus, para a forma da doença AIDS (Ministério da Saúde, 2015).

O desenvolvimento de estratégias preventivas no Brasil, está correlacionado com as ações de testagem e aconselhamento, antes mesmo do surgimento do Sistema Único de Saúde (SUS). Com o advento dos testes rápidos Anti-HIV, o aconselhamento adquiriu um caráter voltado para a aceitação da doença, focando em um suporte emocional, para àqueles pacientes que apresentassem resultados positivos. Contudo, na busca pelo empoderamento dos usuários, dentro da APS, o aconselhamento perpassa pelo meio emocional e transcende o âmbito da testagem, resultando em uma ação em saúde, potencializando ações de prevenção e promoção da saúde, aumentando a autonomia, para ruptura na sucessão do aumento de casos, tanto de HIV, quanto de outras Infecções Sexualmente Transmissíveis (ISTs) (Fonseca \& Iriart, 2012).

O trabalho conjunto das três esferas, nacional, estadual e municipal, ampliam o atendimento, e as necessidades de cada unidade para que não ocorra falta de materiais e insumos necessários para o atendimento de toda a população. A busca ativa, participação e captação dos usuários deve ser incentivada, por toda a equipe de saúde da Estratégia de Saúde da Família, melhorando a abordagem de pacientes com testagem positiva, e acompanhando ativamente o tratamento destes (Silva, Valença, \& Silva, 2017).

O planejamento de ações conjuntas das três esferas políticas, exerce forte influência na realização efetiva dos testes rápidos, tanto pelo fornecimento de subsídios, quanto no incentivo aos profissionais, por capacitações e atualizações constantes. Apesar dos profissionais relatar capacitações presenciais, com o objetivo de implementar e ajudar na realização correta dos testes rápidos, essas capacitações ocorreram de maneira superficial, com foco primordial nos profissionais Enfermeiros e na técnica correta, sem a equipe multiprofissional e com acolhimento, humanização e abordagens fragilizadas, 
bem como, falta de preparo para o aconselhamento especialmente voltado para um paciente com um resultado positivo (Silva, Valença, \& Silva 2017).

"A enfermagem é um importante agente transformador que deve ser capacitado para uma assistência resolutiva na sua pratica diária", tendo sua importância no combate as ISTs, desenvolvendo ações de identificação de vulneráveis, diagnósticos, aconselhamento pré e pós teste, avaliando riscos e agravos ao processo de adoecimento (Oliveira \& Afonso, 2017, p. 01). O acesso universal a informação, referente a transmissão e ao tratamento do HIV, ainda é uma deficiência encontrada no serviço público, onde, especialmente o enfermeiro, tem em sua formação profissional, fortemente evidenciado, a educação em saúde, que busca desenvolver a autonomia na tomada de decisões, e comportamentos saudáveis ao seu contexto (Lima, et al, 2018).

O aconselhamento pré e pós testagem rápida, tanto para os profissionais envolvidos, como para os usuários que vão em busca dos serviços, tem um caráter singular, alternativo e paradoxal, sem desenvolver emoções, vindo ao encontro histórico de prejulgamento, desenvolvido socialmente perante aos usuários que buscam estes serviços. Cabe sublimar que a dimensão emocional e social, permanece uma barreira, no meio acadêmico e prático do profissional, sendo desenvolvido de maneira empírica e própria em cada indivíduo (Neves \& Therense, 2018). Sendo assim, o aconselhamento pré-teste rápido, é um momento de coleta de informações, entrevista, além do preenchimento de documentações (Previati, Vieira, \& Barbieri, 2019), é o momento que se entende o contexto da comunidade e do indivíduo, buscando-se sanar dúvidas e empoderar a população, conforme suas necessidades.

Este estudo se justifica pela necessidade de aconselhamento ao usuário no pré e pós teste rápido anti-HIV, buscando um cuidado integral e acolhedor. Além do mais, frente a um resultado positivo há a necessidade de profissionais capacitados que possam agir da forma correta, indicando soluções passiveis para a melhora do caso. Para tanto, evidencia-se a importância de capacitações constantes, desenvolvendo segurança na revelação do resultado.

O presente trabalho tem por objetivo relatar a experiência de acadêmicas de enfermagem frente à realização de testes rápidos anti-HIV, assim como, a confirmação dos resultados e aconselhamento do usuário.

\section{Metodologia}

Trata-se de um estudo descritivo com abordagem qualitativa, do tipo relato de experiência. O estudo descritivo desenvolve propósitos de observação, descrição e documentação em determinada situação de pesquisa (Polit \& Beck, 2019). A abordagem qualitativa, busca a importância social da pesquisa, o desenvolvimento das relações humanas e sociais, e também a vivência e a experiência de forma objetiva, com utilização de observação, documentação, entrevistas, levando em consideração a ciência e as crenças populares (Minayo, 2009). "Os métodos qualitativos são aqueles nos quais é importante a interpretação por parte do pesquisador com suas opiniões sobre o fenômeno em estudo" (Pereira, Shitsuka, Parreira \& Shitsuka, 2018, p. 67).

$\mathrm{O}$ estudo foi vivenciado por quatro discentes e uma docente do curso de graduação em Enfermagem de uma Universidade privada do Noroeste do Estado do Rio Grande do Sul, durante o estágio curricular da disciplina de Enfermagem no Cuidado a Saúde da Mulher. Ocorreu no mês de abril de 2019, em uma Unidade Básica de Saúde (UBS) de um município do interior do Rio Grande do Sul. A ação contemplou a população adstrita da UBS, tendo como público alvo o atendimento as mulheres, durante as consultas de pré-natal, puerpério, realização do exame clínico das mamas e preventivo do câncer de colo do útero, e realização dos testes rápidos.

\section{Resultados e Discussão}

A ação se deu durante o estágio curricular da disciplina de Enfermagem no Cuidado a Saúde da Mulher, que tem por objetivo: desenvolver através da ação, reflexão e transformação permanente o cuidado de enfermagem às mulheres em relação 
ao gênero, sexualidade e saúde, com interfaces nas questões étnico-raciais e dos direitos humanos; às mães (que vivenciam ou não o processo de nascimento); ao recém-nascido e família, buscando cuidar de forma humanizada.

No decorrer do estágio curricular foram realizados seis testes rápidos anti-HIV, com a presença de um resultado positivo. Os testes rápidos foram desenvolvidos em uma sala da unidade de saúde que se encontrava disponível no momento, em especial, o consultório de enfermagem. Os materiais utilizados para os testes rápidos permaneciam em um armário no consultório de enfermagem, precisando então ser transportado para outro ambiente, em caso de realização em outra sala.

Os testes foram realizados conforme a técnica adequada e seguindo as recomendações do fabricante, sendo utilizado os Equipamentos de Proteção Individual (EPIs). Em seguida foram conferidas as orientações aos usuários, além de aconselhamento eficiente pré e pós teste e necessidade de boa comunicação entre os profissionais da equipe da Unidade Básica de Saúde (UBS) e os profissionais da rede. Para o caso do diagnóstico de HIV positivo, foi necessária aplicação de novo teste rápido de confirmação, para assim encaminhar a usuária ao Serviço de Atenção Especializada (SAE).

Na ocasião a jovem, procurou a unidade de saúde para realização do exame preventivo do câncer de colo do útero e de mama, partindo de sua iniciativa a realização do teste rápido. Relatou que havia realizado o teste há seis meses, e que procurava a unidade de saúde regularmente para tal, porém, os resultados anteriores foram não reagentes. Durante a realização do teste rápido anti-HIV, identificou-se certa apreensão com relação ao resultado. Após resultado positivo em primeiro teste rápido anti-HIV, a usuária apresentou-se chorosa e surpresa com o resultado. Buscou-se tranquilizar a usuária, para realização do segundo teste rápido anti-HIV, necessário para diagnóstico. Torna-se fundamental que o Segundo Teste rápido tenha uma especificidade igual ou superior ao primeiro teste, com objetivo de minimizar desconfortos em caso de falso-positivo (Ministério da Saúde, 2016). Após realização e confirmação do diagnóstico, usuária continuava chorosa e inconformada com o resultado positivo. Diante da situação, percebeu-se a insegurança e falta de informação da usuária com relação ao tratamento, e o medo de julgamento por parte dos familiares e sociedade.

Embora, o teste rápido para deteç̧̃ao do vírus HIV, desenvolve uma série de medos e inseguranças em cada paciente, o enfermeiro tem papel indispensável no aconselhamento, transformando esse momento mais seguro, transmitindo apoio e acolhimento. É um momento de mostrar quais são as alternativas e instruções que devem ser seguidas, em caso de testagem positiva (Oliveira \& Afonso, 2017). Em meio ao desenvolvimento do Sistema Único de Saúde o aconselhamento busca desenvolver o cuidado integral dos sujeitos, "o aconselhamento é uma ferramenta que visa a promoção e prevenção da saúde por meio de um momento de conversa antes da realização do teste e outro pós-teste, para a devolução do resultado" (Rocha, Santos, Conz, Silveira, 2016, p. 27), é uma ferramenta complexa e utilizada principalmente para a vinculação do usuário e essencial para o cuidado (Rocha, Ew, Moro, Zanardo \& Pizzinato 2018).

O aconselhamento é um momento único vivenciado e construído a partir das expectativas, medos e inseguranças de cada paciente, não é um procedimento e deve ser realizado com o outro, com motivação e interesse da pessoa que está sendo atendida. Possui uma "Dimensão Educativa, Dimensão da Avaliação de Risco e Dimensão do Apoio Emocional”, e a partir disto deve-se reduzir o impacto dos diagnósticos, identificar as vulnerabilidades e as medidas preventivas, além do próprio risco (Melo, 2018, p. 06; Rocha, et al., 2016).

Diante da presença de um teste rápido positivo verificou-se a importância de realizar acolhimento e aconselhamento com todos os usuários, independentemente de eles terem ou não realizado teste rápido anteriormente. Percebe-se que, apesar de toda a capacitação profissional recebida, frente a um caso de testagem positiva acabam surgindo dúvidas e inseguranças, tanto pelo usuário que se sente impotente frente o ocorrido, quanto por parte do profissional que se vê comovido pela situação enfrentada. Além disso, verifica-se a necessidade de explicação antes da realização do teste, utilizando este tempo para educação em saúde, onde evidencia-se formas de prevenção, tratamento e prognóstico da doença. A implementação, 
divulgação e realização dos testes rápidos na população, acabou tendo um propósito de orientação e acompanhamento do usuário, empoderando-o a ser o protagonista de sua saúde, apesar desta ser ainda uma deficiência na assistência.

Um estudo que teve por objetivo a realização de ações de aconselhamento do teste rápido de HIV na atenção básica, trouxe como resultados que os profissionais com ensino superior apesar de serem capacitados não realizam o teste rápido, cabendo ao enfermeiro tal atribuição. Os profissionais relatam que tem consciência da importância da realização do aconselhamento pré e pós teste rápido, mas os enfermeiros referem que a sobrecarga de trabalho, o aumento na procura dos serviços, e a infraestrutura precária, sem lugar específico para realização dos testes, acabam prejudicando a realização destes, de forma correta, prejudicando o acolhimento e aconselhamento do usuário (Rocha, et al., 2018). "O aconselhamento bem feito, realizado por um profissional bem capacitado, pode aumentar as possibilidades do usuário atendido, se implicar mais efetivamente com as medidas necessárias para a prevenção das IST/HIV/Aids" (Dutra, 2020, p. 21).

A relação dos profissionais enfermeiros com a revelação do diagnóstico positivo se apresenta como uma dificuldade enfrentada no campo profissional. $\mathrm{O}$ enfermeiro tem a responsabilidade técnica pelo teste rápido e deve estar preparado para ambos os resultados, realizando a aproximação, transmissão de segurança e acolhimento adequadas do paciente, em meio a qualquer angústia (Araújo, Quirino, Pinho \& Andrade, 2018). O aconselhamento pós testagem positiva deve ser realizada respeitando-se cada paciente e observando que cada pessoa receberá má notícias de uma maneira diferente. O profissional deve manter um ambiente adequado, confortável, olhar nos olhos do paciente e ter empatia e humanização ao dar uma má notícia, sempre responder as suas dúvidas e manter uma educação continuada, preferencialmente com uma equipe multiprofissional (Almeida \& Bonamigo, 2017).

A efetividade na aplicação e diagnóstico dos testes rápidos, leva a um melhor prognóstico da doença, e consequente promoção da saúde dos envolvidos. O trabalho em equipe bem desenvolvido, de forma interdisciplinar, com metodologias conjuntas e encontros de capacitação constantes, favorece a troca de experiências e conhecimentos gerando consequente evolução no desenvolvimento da assistência (Moraes \& Nascimento, 2016).

Uma revisão integrativa que trouxe à tona as produções que evidenciavam o cuidado e o acolhimento do enfermeiro, no caso de pacientes com HIV, apresentou como conclusão que o acolhimento pode gerar, se bem realizado, um ambiente de prevenção e proteção à saúde, principalmente agravos e situações evitáveis. No entanto, como relatado neste estudo, o acolhimento não é o momento para se realizar educação em saúde para o desenvolvimento de "consciência crítica", mas sim, um momento de aproximação, onde o usuário tenha confiança de relatar sua angústia, medos e dificuldades para o "fortalecimento do processo formativo", considerando e respeitando a individualidade de casa sujeito (Veras, et al, 2018, p. 1206).

Cabe ressaltar que, o enfrentamento de um resultado positivo, gera desconfiança e insegurança por parte do usuário, principalmente pela falta de conhecimento e julgamentos que ocorrem em toda a sociedade. Mesmo após tantos avanços com relação ao tratamento da doença aids, proveniente do vírus HIV, por meio da utilização de antirretrovirais, cada vez menos danosos ao organismo, ainda há muitos estigmas presentes na sociedade, o que leva a um descaso pelos testes rápidos, devido a insegurança na presença do resultado. Sendo assim, percebe-se a necessidade cada vez maior de desenvolvimento de ações que ressaltem a importância do diagnóstico e tratamento adequados (Araújo, et al, 2018).

O enfermeiro ainda é o principal profissional na realização dos testes rápidos, o que acaba muitas vezes prejudicando a eficiência da assistência, já que, demanda tempo e aprendizagem constante do profissional, e este muitas vezes, se sente sobrecarregado pela grande demanda de usuários e pela falta de apoio da gestão. Provocando assim, o descaso pelo aconselhamento pré e pós teste, banalizando este momento de educação em saúde, onde o usuário se apresenta acessível, permitindo a transmissão de informações referentes a métodos de prevenção, diagnóstico e tratamento do HIV, contribuindo para a redução da exclusão social de pessoas com HIV. 
Estudo que buscou abordar a relevância do profissional enfermeiro na ampliação do acesso aos testes rápidos e ao aconselhamento, reforçar a necessidade de oferta para toda a população, ampliação de testes rápido nas Unidades Básicas, oferecendo-os para todos usuários. O estudo ainda esclarece que um paciente frente a um resultado positivo, que foi devidamente acolhido, orientado e aconselhado, terá uma visão diferente sobre os métodos de tratamento e consequente aumento na expectativa de vida, já que se sentirá mais seguro e apoiado pelo profissional (Oliveira \& Afonso, 2017).

\section{Considerações Finais}

A realização dos testes rápidos nas UBS tem grande relevância, já que aproxima a população de um acompanhamento adequando de sua saúde. O presente trabalho demonstrou a necessidade de uma comunicação e aconselhamento de maneira segura e eficiente, antes e após a realização dos testes rápidos para HIV. A vivência de um resultado positivo provocou mudança de visão e pensamentos, onde percebeu-se que há a necessidade de um trabalho em rede, com a disponibilidade de múltiplos profissionais para um cuidado integral e efetivo. Para tanto, um aconselhamento efetivo, anterior a realização do teste, é uma forma de conscientizar o paciente e prepará-lo para ambos os resultados, e este não pode ser simplesmente omitido de nossa assistência. A busca constante de conhecimento e atualizações desenvolve e capacita o profissional para a responsabilidade de comunicar o resultado e realizar o diagnóstico, demonstrando soluções existentes e promovendo o acolhimento compatível com suas necessidades para manter a saúde e a dignidade humana.

Considera-se como limitação deste estudo a realização de somente seis testes, sendo que, apenas um teve um resultado positivo, além de serem realizados unicamente em uma unidade de saúde. Futuramente, sugere-se a realização de novos estudos, em uma população em maior quantidade, com profissionais capacitados e que saibam o fluxo correto a seguir na assistência, independente do resultado encontrado.

\section{Referências}

Almeida, M. C., \& Bonamigo, E. L. (2017). Como dar más notícias: diagnóstico de HIV por meio do teste rápido. Anais Da Semana Acadêmica E Mostra Científica De Enfermagem, 10. https://unoesc.emnuvens.com.br/anaissamcenf/article/view/12544

Araújo, W.J. Quirino, E. M. B., Pinho, C. M., \& Andrade, M. S. (2018). Percepção de enfermeiros executores de teste rápido em Unidades Básicas de Saúde. Rev Bras Enferm, 71(Suppl 1), 631-636, https://doi.org/10.1590/0034-7167-2017-0298.

Dutra, M. S. (2020). Aprimoramento do Aconselhamento Pré e Pós Teste Hiv/Sífilis/Hepatite B e C na Atenção Básica de Saúde de Betim. Trabalho de Conclusão de Curso - Especialista em Saúde Pública. Escola de Saúde Pública, Minas Gerais, Brasil. http://repositorio.esp.mg.gov.br:8080/xmlui/bitstream/handle/123456789/369/TCC\%20Mariela\%20Santiago\%20Dutra.pdf?sequence=1\&isAllowed=y

Fonseca, P. L. \& Iriart, J. A.B. (2012). Aconselhamento em DST/Aids às gestantes que realizaram o teste anti-HIV na admissão para o parto: os sentidos de uma prática. Interface - Comunic., Saude, Educ., 16(41), 395-407. https://doi.org/10.1590/S1414-32832012000200009

Lima, A. C. M. A. C. C. et al. (2018). Tecnologias e práticas educativas para prevenção da transmissão vertical do HIV. Rev Bras Enferm. 71(suppl 4):18621871. https://doi.org/10.1590/0034-7167-2016-0333

Mello, A. M. (2018). Acolhimento, Aconselhamento e Orientações Pré e Pós-Teste. Ministério da Saúde. 2018.17 slides. http://www.aids.gov.br/system/tdf/pub/2016/65618/dia_9_8_-_acolhimento_e_aconselhamento_ana_monica.pdf?file=1\&type=node\&id=65618\&force=1.

Minayo, M. C. S. (2009). Pesquisa social: teoria, método e criatividade. $28^{\mathrm{a}}$ ed. Petrópolis: Vozes.

Ministério da Saúde. Secretaria de Vigilância em Saúde. (2015). Protocolo Clínico e Diretrizes Terapêuticas (PCDT). Atenção integral as pessoas com infecções sexualmente transmissíveis (IST). 2.ed. Brasília: 2015. http://www.aids.gov.br/pt-br/pub/2015/protocolo-clinico-e-diretrizes-terapeuticas-paraatencao-integral-pessoas-com-infeccoes

Ministério da Saúde. Secretaria de Vigilância em Saúde. Departamento de Vigilância. (2016). Prevenção e Controle das Infecções Sexualmente Transmissíveis, do HIV/Aids e das Hepatites Virais. Manual Técnico para o Diagnóstico da Infecção pelo HIV em Adultos e Crianças. Brasília: 2016. http://www.aids.gov.br/pt-br/node/57787

Moraes, J. T. \& Nascimento, R. L. F. (2016). Planejamento estratégico e implantação dos testes rápidos de HIV, Sífilis e Hepatites virais em uma capital brasileira: relato de experiência. Rev Bras Promoç Saúde. Fortaleza, 29(1): 139-44. https://doi.org/10.5020/18061230.2016.p139

Neves, A. L. M. \& Therense, M. (2018). Hiv/Aids, gênero e sexualidade: políticas e práticas de prevenção, testagem e Aconselhamento. Manaus (AM): Editora UEA. 
Research, Society and Development, v. 10, n. 7, e32310716489, 2021

(CC BY 4.0) | ISSN 2525-3409 | DOI: http://dx.doi.org/10.33448/rsd-v10i7.16489

Oliveira, J. A. S. \& Afonso, T. M. (2017). O Teste rápido para o Diagnostico de HIV na Atenção Primária à Saúde e a importância da atuação do Enfermeiro. Congresso Internacional de Enfermagem, Universidade Tiradentes, Aracajú, SE, Brasil.

Pereira, A.S. Shitsuka, D.M., Parreira, F.J. \& Shitsuka, R. (2018). Metodologia da pesquisa científica. 1. ed. Santa Maria, RS: UFSM, NTE, 2018.

Polit, D. F. \& Beck, C. T. (2019). Fundamentos de pesquisa em enfermagem: avaliação de evidências para a prática da enfermagem. 9. ed. Porto Alegre: Artmed.

Previati, S. M., Vieira, D. M. \& Barbieri, M. (2019). A importância do aconselhamento no exame rápido de HIV em gestantes durante o pré-natal. J. Health Biol Sci. 7(1), 75-81. http://dx.doi.org/10.12662/2317-3076jhbs.v7i1.2104.p75-81.2019

Rocha, K. B., Santos, R. R. G., Conz, J., Silveira, A. C. T. (2016). Transversalizando a rede: o matriciamento na descentralização do aconselhamento e teste rápido para HIV, sífilis e hepatites. Saúde Debate. Rio de Janeiro, 40(109), 22-33. DOI: 10.1590/0103-1104201610902

Rocha, K. B., Ew, R. A. S., Moro, L. M., Zanardo, G. L. P. \& Pizzinato, A (2018). Aconselhamento na perspectiva de profissionais da atenção básica: desafios na descentralização do teste rápido HIV/Aids. Ciencias Psicológicas. 12(1): 67 - 78. doi: 10.22235/cp.v12i1.159

Silva, I. T. S., Valença, C. N., \& Silva, R. A. R. (2017). Cartografia da implementação do teste rápido anti-HIV na Estratégia Saúde da Família: perspectiva de enfermeiros. Esc Anna Nery. 21(4), 01-08. https://doi.org/10.1590/2177-9465-ean-2017-0019

Silva, J. V. F., Júnior, F. J. M. do N., \& Rodrigues, A. P. R. A. (2014). Fatores de não adesão ao tratamento antirretroviral: desafio de saúde pública. Caderno De Graduação - Ciências Biológicas E Da Saúde - UNIT - ALAGOAS, 2(1), 165-175. Recuperado em 14 de março de 2021 de https://periodicos.set.edu.br/fitsbiosaude/article/view/1193

Veras, S. M. J. et al. (2018). Reflexões sobre Cuidado e Aconselhamento de Profissionais de Saúde e Enfermeiros Em HIV/AIDS nos Centros de Testagem e Aconselhamento: Revisão Integrativa. Id on Line Rev. Mult. Psic., 12(40), 1193-1208. https://doi.org/10.14295/idonline.v12i40.1123 